\title{
Driving Simulators for Human Vehicle Interaction Design
}

\author{
Alexandra TUCĂ \\ Scientific Research and Continuous Training Center for Sustainable Automotive Technologies, \\ University Politehnica of Bucharest, Romania \\ srctcsat@upb.ro \\ Valerian CROITORESCU \\ Scientific Research and Continuous Training Center for Sustainable Automotive Technologies, \\ University Politehnica of Bucharest, Romania \\ valerian.croitorescu@upb.ro

\section{Mircea OPREAN} \\ Scientific Research and Continuous Training Center for Sustainable Automotive Technologies, \\ University Politehnica of Bucharest, Romania \\ mircea.oprean@upb.ro

\section{Thomas BRANDEMEIR} \\ Center of Automotive Research on Integrated Safety Systems and Measurement Area, Technical \\ University of Ingolstadt, Germany \\ thomas.brandmeier@thi.de
}

\begin{abstract}
The interaction human-vehicle, as well as driver's behavior are subject long debated in the automotive engineering domain. Driving simulators have an extraordinary important role allowing research that would not be possible to study in real world scenarios.

A driver uses his sensory inputs to obtain the required input to base his decision on. The bandwidth of the required input signal should be in accordance to the driver's task. For simple tasks, like turning on the screen wipers or direction indicator, low frequency information is sufficient. High frequency information is required when cornering on a busy road or when driving in relatively limit situations.

The optimal configuration of each sub-system remains a significant cause for debate and still poses a major challenge when considering the ability of simulators to extract realistic driver behavior. If a difference is observed between real and virtual conditions, the factors specifically cause these differences are very difficult to be explained.
\end{abstract}

Keywords: human vehicle interaction, driver behavior, vehicle control design, virtual vehicle environment. 


\section{INTRODUCTION: MOTION SIMULATION AND THE NEED FOR MOTION}

Simulation is defined as the imitation of reality. Motion simulation is all about perception. Motion perception for the human body has two inputs: inertial stimulants on the body and environmental motion with respect to the body. The vestibular system located in the inner ear is the prominent sense that provides the perceptual system with the information about linear and angular inertial acceleration of the body. The speed prominent sense for the human body is provided through the visual system.

There are several reasons why a motion system is important in simulation, the main reason being to prevent driving simulator sickness. Symptoms of simulator sickness come from the differences between the latency of the visual and motion system and false cues.

The driver uses his/her sensory inputs to obtain the required input to base his/her decision on. The bandwidth of the required input signal should be in accordance to the driver's task. For simple tasks, like turning on the screen wipers or direction indicator, low frequency information is sufficient. High frequency information is required when cornering on a busy road or when driving in relatively limit situations.

\section{DRIVING SIMULATORS PERFORMANCES}

\section{Versatility and Development}

The main advantage of research simulators is the versatility. They can be easily and economically configured to simulate a variety of human factors research problems. They allow evaluation and optimization of human performance within system constraints and indicate problem areas in system design and functioning. They are particularly useful in selecting a viable system approach from numerous alternatives and evaluating system performance before field testing. Different simulation scenarios can be created to match the requirements of the particular experiment. Environmental effects such as foggy roads, snowy or slippery roads or night time driving conditions can be created. Vehicle characteristics can be altered quickly - steering rations, spring rates, damping factors, driven wheels. New roadways or infrastructure can be created in the driving simulator where the test situation is difficult to be reproduced on the road. They can often represent the most cost-effective approach in a given application. Complete instrumentation and recording systems for in-vehicle tests can be expensive to set up and maintain. Contriving typical traffic situations, including interactive vehicles and signal controls, on special driving courses can also be costly. On the other hand, it is often less expensive to set up and operate simulations in a controlled laboratory environment than it is to conduct field tests that are designed to achieve given experimental objectives. In particular, stimuli and events external to the driver's vehicle are substantially cheaper to implement, control and vary in a simulator that they are on a test track. 


\section{Experimental Control and Measurement}

Driving simulators are able to control experimental conditions over a wider range than field tests and can be easily changed from one condition to another. This capability can be important in terms of experimental design characteristics, such as allowing back-to-back comparisons of disparate experimental conditions. Every driver can drive the exactly same testing situation where systematic variation in road, vehicle or traffic situations conditions is difficult to achieve in real world. Criterion variables can easily be made available in a driving simulator. Performance measures can be easily mechanized. Digital computer systems can further provide on-line data processing, formatting and storage and the reduction and compact arrangement of data.

\section{Safety}

Driving simulators provide an inherently safe environment for driving research. There is no endangerment to the driver or other road users under critical driving conditions or when testing innovative in-vehicle devices. They can be used where approval for an on-road experiment is unlikely to be forthcoming from the relevant authorities without some prior evidence on behavioral and safety issues.

\section{Validity}

The major disadvantage of driving simulators used for research is represented by the fact that the real world will never be replicated in all its complexity. There will always be the issue of validity, of to what extent behavior in a simulator corresponds to real life.

\section{Costs}

Driving simulators have a high initial acquisition cost. In addition, operating and maintenance costs are slightly higher than for training simulators because research simulators are more complex.

\section{Simulation Sickness}

Simulation sickness can vary widely among individuals who experience it among simulators that induce it. Effects may range from mild disorientation and queasiness to sever ataxia and full emesis. The most critical variables are the visual horizontal field of the view and the level of moving scene detail. The potential simulator design etiological factors summarized by Casali and Wierwille (1986) can be:

a. Control loop lags and delays. Inappropriate control-feedback lags are known to degrade controllability and stability of vehicular systems, as demonstrated may introduce symptoms of sickness;

b. Dynamic characteristics like proper modelling of the full-scale vehicle in the simulator's computational systems and accurate correspondence between the simulator's dynamics and those of the actual vehicle (both for low-speed maneuvers and more complex high-speed kinematics);

c. Control load factors like damping;

d. Motion system factors. Barrett and Thornton (1968) suggested that fixed base simulators are likely to induce sickness because a cue conflict arises when the operator visually sense the appearance of incident vehicular motion but never receives corresponding physical acceleration or positional cues. On the other 
hand, the addition of motion cueing systems to some simulators has greatly reduced the sickness problem;

e. Visual system factors such the display type, the field-of-view and scene detail, the display rate and the display distortions;

f. Cockpit environment factors such as temperature regulation and humidity control when the simulator cab is enclosed.

\section{SIMULATION OF THE ROAD ENVIRONMENT AND REALISM}

The simulation of the road environment is very complicated because the more details are provided the more slowly the simulator will run. However, since a certain driving pattern may not be replicated perfectly within a driving condition, the consistency in the driving pattern between different trials within each of the driving conditions (simulator vs. real world) has to be estimated first. In several studies, the question "How realistic do you think the driving in the simulator was?" has been asked. Comparing the results from different studies, the lowest realism and also some "not at all realistic" ratings appear in the studies of anti-collision and vision enhancement systems. These systems are not in common use yet and may add unrealism to the situation. Also, the fact that 5\% of the elderly estimated the driving to be "not at all realistic" in a study concerning the effects of mobile phone use on elderly drivers may very well reflects that using a mobile phone is an unrealistic task for this section of population.

\section{SYSTEM ARCHITECTURE}

The architecture contains the vehicle mock-up and its platform, the hexapod including the hydraulic system, the hydraulic power unit and the steel plated needed for the load distribution, the video and the audio systems and the computer cabinet. All the components are part of two different global systems: the control system and the mock-up system (Figure1).

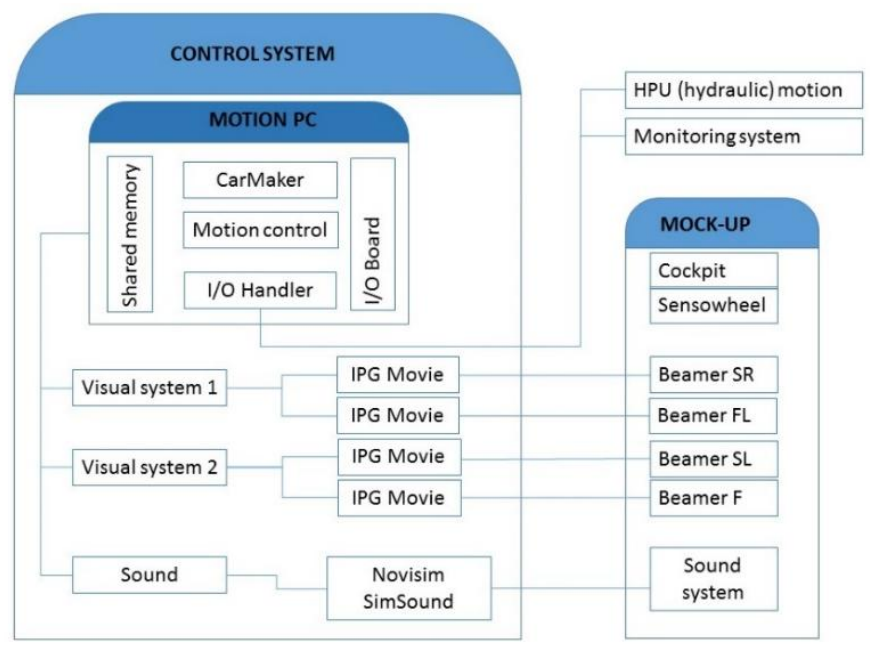

Figure 1: System architecture 
The vehicle mock-up is an actual vehicled modified to have the same characteristics and parameters with the real vehicle, in respect with the task requirements. The platform is used to fix the vehicle with the hexapod. The motion system consists of a classical Steward hexapod with 6 hydraulic actuators that make the motion possible on 6 degrees of freedom. The actuators receive the motion commands via the hydraulic system that is operated from the motion unit. The steel plates for the load distribution are the contact with the ground that assures the stability of the motion platform.

The visual system consist in several projectors: placed in front and 2 in lateral views that displays different resolution images. The audio system is incorporated in the simulator cabin: in front side speakers are mounted below the instrument panel in the footwall of the driver / passenger, while the subwoofer, center speaker and two rear speakers are located in the rear cabin section. The audio and the video systems have to reproduce with high fidelity the real environment while vehicle is running.

\section{KEY SYSTEMS OF A RESEARCH DRIVING SIMULATOR}

\section{Scenario Control}

The scenario control refers to the process of choreographing particular traffic scenarios or events within the virtual driving environment. It achieves this through a modification of the scene-graph to add all the real-time agents based on a model of their behavior, such as other vehicles, pedestrians or traffic lights. Fundamental to scenario control is the underlying description of the roadway, the Logical Road Network - LRN. The scenario control uses the LRN to provide information in order to support the behavior and the interaction of the real-time agents. For example, intelligent virtual traffic effectively uses the LRN to "perceive" the road as a human driver in order to make intelligent decisions such as intersection priorities and overtaking.

\section{Driver/Vehicle Data Processing}

One of the fundamental reasons for using a research driving simulator is the abundance of driver behavioral and performance measures that can be easily recorded. These data may refer to the driver's use of the vehicle controls, the corresponding behavior of the vehicle or specific behavioral metrics that are commonly used to quantify driver behavior, such as coherence in a car following task (Brookhuis, de Vries, \& de Waard, 1991), steering reversal rate (McLean \& Hoffmann, 1975) or time- to-line crossing (Godthelp \& Konnings, 1981).

\section{Control Loading}

The "feeling" for a simulated drive helps to create a sense of realism in a driving simulator; therefore, it would be convenient that the vehicle controls have the same characteristics as the actual vehicle that the simulator is mimicking. The main feedback to a simulator driver is through the steering emanating from those generated at the tyre-road interface as modeled by the vehicle dynamics. Control feedback through the foot pedals is also significant. 


\section{Motion System}

A motion system is designed to artificially recreate the dynamic cues of both longitudinal (braking and ride) and lateral (cornering and stability) vehicle accelerations. Dynamic cueing in a driving simulator is possible using motion platforms which were initially developed for flight simulation applications, progressively used more frequently in the automotive field. Developments in vehicle simulation applications started relatively recently (Nordmark, Lidström \& Palmkvist, 1984; Drosdol \& Panik, 1985) compared to the initial hexapod design used in early flight simulators (Stewart, 1965).

\section{EFFECTS OF THE MAIN SYSTEMS ON DRIVING SIMULATOR VALIDITY}

Three main modalities through which drivers sense their movement within the virtual environment are contained into a research driving simulator: stimulation of the visual system, the vestibular system and via auditory information (Kemeny \& Panerai, 2003). It has been for some time commonly regarded that the visual, and to a lesser extent the vestibular feedback are the most important with regard to the perception of vection. Recently the accuracy of audio rendering has been shown to influence such vection. Although, its effect is much weaker and typically only occurs in between $25 \%$ and $60 \%$ of people (Sakamoto, Osada, Suzuki \& Gyoba, 2004).

\section{Effects of the Visual System}

Under natural conditions, visual cues provide a significant contribution to allow an observer to form a perception of their environment space. However, under simulated conditions, the inferior display characteristics (e.g. image resolution, update frequency and field of view) bring a quality reduction of these cues. The driver's use of these cues is important for the estimation of: vehicle speed, the distance to objects and vehicle heading and lateral control.

The evaluation of vehicle speed and the estimation of inter-vehicle distance are essential skills in safe and controlled driving. Maneuvers such as overtaking and collision avoidance require such abilities. These skills require the accurate representation of self-motion from both optic flow and egocentric direction - the direction of an object in space relative to the observer (Gogel \& Tietz, 1979). Optic flow can give information about either absolute speed or distance and also exploited to compare relative spatial intervals, central to the accurate estimation of time-to-contact (Lee, 1976). A significant number of studies into speed perception have shown that observers tend to underestimate their velocity in simulated environments (Alicandri, Roberts \& Walker, 1986; Riesmersma, van der Horst \& Hoekstra, 1990; Duncan, 1995; Groeger, Blana, Carsten \& Jamson, 1999).

Distance estimation is also based on a number of reliable cues, such as optic flow (Bremmer \& Lappe, 1999), disparity (Howard \& Rogers, 1995) and motion parallax (Rogers \& Graham, 1979). Motion parallax describes the differential motion of pairs of points as a result of their different depths relative to the fixation point and to the motion of the observer. It provides robust estimation of absolute egocentric distance when combined with additional visual information describing an observer's self-motion. 


\section{Effects of the Motion System}

The vection usually takes several seconds to establish itself (Melcher \& Henn, 1981) in a stationary observer. The latency of this vection can be reduced by the addition of inertial motion cues (Groen, Howard \& Cheung, 1999). Furthermore, from a steady condition of stabilized speed and lane position, drivers are experiencing a disturbance to such conditions. They exhibit a significantly shorter response time in simulators with motion as opposed to without (Wierwille, Casali \& Repa, 1983).

Greater variation in lane position has been observed in drivers of fixed-base simulators compared to those experiencing similar but real-life conditions (Duncan, 1995; Blana \& Golias, 2002). The addition of motion cues reduces this variation (Alm, 1995; van Winsum \& Godthelp, 1996; Reymond, Kemeny, Droulez \& Berthoz, 2001; Greenberg, Artz and Cathey, 2002). Moreover, drivers perform wider turns when lateral cues are present compared to those when only visual information is available (Siegler, Reymond, Kemeny \& Berthoz, 2001).

\section{Effects of the Sound System}

Compared to the visual inducement of self-motion, audio cues are much less compelling. Auditory vection is influenced by the realism of the acoustic simulation and the number of sound sources.

Sound cues are so frequently represented in driving simulation that unearthing a driving simulator without the provision of a sound system would be quite a discovery. However, the effect of audio cues on the fidelity of simulator driver behavior in comparison to real conditions has not been shown so clearly. McLane and Wierwille (1975) investigated the effects of presence or absence of speed-related sounds and vibrations in a driving simulator. The results indicated that the performance measures of yaw, lateral and velocity deviation were significantly affected by the deletion of vibration. The authors reported that the existence of audio had no significant effect on either driving speed or lane control. However, they acknowledged that the audio rendering had the advantage that irrelevant sounds emanating from the various simulator sub-systems were effectively masked, improving the simulator's face validity. Similarly, some twenty years later, Davis and Green (1995) confirmed the lack of an effect of sound in a simple fixed-base simulator, demonstrated by unchanged drivers' rating of realism with and without audio cues, a result replicated by Capustiac, Hesse, Schramm \& Banabic (2010).

\section{CONCLUSION}

The present study highlights the important role of driving simulators in multiple research areas. Furthermore, this study indicates that there are benefits to providing drivers with the opportunity to practice improving upon their driving techniques in a driving simulator.

The presented approach and aspects regarding the driving simulation have high importance in terms of the motion system, preliminary results before field testing, developing new very hard to be reproduced in real world scenarios, safety environment while entire driving simulation process, high fidelity while reproducing real world, very close to all its 
complexity, and last, but not least, the costs effective by using driving simulators than field testing.

The human-vehicle interfaces are trying to find the optimal configuration of each subsystem. But it remains a significant cause for debate and still poses a major challenge when considering the ability of simulators to extract realistic driver behavior. The effects from the main key systems are very important on evaluating the human-vehicle communications. If a difference is observed between real and virtual conditions, the factors specifically cause these differences are very difficult to be explained.

This study leads to ideas on how to optimize the driving simulator behavior and the human to vehicle communications. The interaction between users and vehicles will be long debated subject. New further researches are needed in order to achieve the control measures for the gaps between the level based functioning and the level specific to each user demands.

\section{ACKNOWLEDGEMENT}

This paper represent part of the work done during the first author research stage for her Engineer Diploma, under the supervision of As. Prof. Valerian Croitorescu and Prof. Thomas Brandmeier, inside Driving Simulations Laboratory, from Zentrum für Angewandte Forschung Ingolstadt. This paper is included in a series of scientific papers found in the references, presenting some of the authors' research activities on driving simulation.

\section{REFERENCES}

Alicandri, E., Roberts, K., \& Walker, J., (1986) A validation study of the DoT/FHWA simulator (HYSIM)(FHWA/RD-86/067), Washington, DC, US Department of Transportation, Federal Hyghway Admnistration;

Alm, H., (1995), Driving simulators as research tools - a validation study based on the VTI driving simulator, Unpublished, Internal report VTI, Linkoping Sweden, VTI, wedish National Road and Transport Research Institute;

Barrett, G., \& Thornton, C.L., (1968), Relationship between perceptual style and simulator sickness, Journal of Applied Psyhology, 52, 304-308 (a)

Barrett, G., \& Thornton, C.L., (1968), Relationship between perceptual style and driver reaction to an emergency situation, Journal of Applied Psyhology, 2, 169-176 (b)

Blana, E., \& Golias, J., (2002), Differences between vehicle lateral displacement on the road and in a fixed-base simulator, Human Factors, 44, 303-313

Bremmer, F., \& Lappe, M., (1999), The use of optical velocities for distance discrimination and reproduction during visually simulated self-motion, Experimental Brain Research, 127 (1), 33-42

Brookhuis, K.A., de Vries, G., \& de Waard, D., (1991), The effects of mobile telephoning on driving performance, Accident Analysis and Prevention, 23, 309-16 
Capustiac, A., Hesse, B., Schramm, D., \& Banabic, D., (2010), A Human Centered Control Strategy for a Driving Simulator International Journal of Mechanical \& Mechatronics Engineering, IJMME-IJENS Vol: 11 No: 01, 2011

Casali, J.G., \& Wierwille, W.W., (1986), On the measurement of pilot perceptual workload: A comparison of assessment techniques addressing sensitivity and intrusion issues, Ergonomics, 27, 1033-1050

Davis, B.T., \& Green, P., (1995), Benefits of sound for driving simulation: An experimental evaluation (Tech.Rep. UMTRI-95-16), Ann Arbor, MI: University of Michigan

Duncan, B., (1995), Calibration trials of the TRL driving simulator (TRL report, PA/3079/95), Crowthrone, England, Transport Research Laboratory

Drosdol, J., \& Panik, F., (1985), The Daimler-Benz driving simulator a tool for vehicle development, SAE Paper, 850334, Society of Automotive Engineers International Congress and Exposition, Detroit, MI

Godthelp, H., \& Konnings, H., (1981), Levels of steering control; some notes on the time-to-line-crossing concept as related to driving strategy, 1st European annual Conference on Human Decision - Making and Manual Control, Delft

Gogel, W,C., \& Tietz, J.D., (1979), A comparasion of oculomotor and motion parallax cues of egocentric distance, Vision Research, 19(10), 1161-1170

Greenberg, J., Artz, B., \& Cathey, I., (2002), The effect of lateral motion cues during simulated driving, Proceedings of the Driving Simulation Conference, North America, 2003, Dearborn, MI

Groeger, J.A., Blana, E., Carsten, O., \& Jamson, H., (1999), Speed and distance estimation under simulated conditions, in Gale, A. (Ed.), Vision in Vehicles VII, 291-300

Groen, E.L., Howard, I.P., \& Cheung, S.K., (1999), Influence of the body roll on visually induced sensation of self-tilt and rotation, Perception 28(3), 287-297

Howard, I.P., \& Rogers, B.J., (1995), Binocular vision and stereopsis, Oxford University Press

Kemeny, A., \& Panerai, F., (2003), Evaluating perception in driving simulation, Trends in Cognitive Sciences, 7(1), 31-37

Lee, D.N., (1976), A theory of visual control of braking based on information about time-to-collision, Perception, 5(4), 437-459;

McLane, R.C., \& Wierwille, W.W., (1975), The influence of motion and audio cues on driver performance in an automobile simulator, Human Factors, 17(5), 488-501

McLean, J.R. \& Hoffmann, R. (1975), Steering reversals as a measure of driver performance and steering task difficulty, Human Factors, 17(3), 248-256

Melcher, G.A., \& Henn, V., (1981), The latency of circular vection during different accelerations of the optokinetic stimulus, Perception and Psychophysics, 30(6), 552-556

Nordmark, S., Lidström, M., \& Palmkvist, G., (1984), Moving base driving simulator with wide angle visual system, SAE Tech Paper 845100, Warrendale, PA: Society of Automobile Engineers;

Reymond, G., Kemeny, A., Droulez, J., \& Berthoz, A., (2001), Role of lateral acceleration in curve driving: Driver model and experiments on a real vehicle and a driving simulator, Human Factors, 43 (3), 483-495 
Riemersma, J., Van der Horst, R., Hoekstra, W., et al. (1990). The validity of a driving simulator in evaluating speed reducing measures. Traffic Engineering and Control, 31(78), 416-420

Rogers, B., \& Graham, M., (1979), Motion Parallax as an Independent Cue for Depth Perception, Perception 8, 125-34

Sakamoto, S., Osada, Y., Suzuki, Y., and Gyoba, J., (2004), The effects of linearly moving sound images on selfmotion perception, Acoustical Science and Technology 25, 100-102

Siegler, I., Reymond, G., Kemeny, A., \& Berthoz, A., (2001), Sensorimotor integration in a driving simulator: Contribution of motion cueing. Elementary driving tasks, Proceedings of driving simulation conference, 21-32

Stewart, D., (1965), A platform with six-degrees-of-freedom, Proceedings of the Institution of Mechanical Engineers, 180 (Part 1, 5), 371-386

Tucă, A., Croitorescu, V., Brandmeier, T., (2015) Driving Simulator Development: Phase I - From State of Art to State of Work, Proceedings of Industrial Simulation Conference, Universidad Politecnica de Valencia, Spain

Tucă, A., Croitorescu, V., Brandmeier, T., (2015), Driving Simulator Development Phase II - Building and Controlling Scenarios through the Motion Algorithm, Proceeding of European Simulation and Modelling Conference, UK

Van Winsum, W., Godthelp, H., (1996), Speed Choice and Steering Behaviour in Curve Driving, Human Factors Vol. 38, lssue 3, S. 434-441

Wierwille, W. W., J. G. Casali \& B. S. Repa (1983), Driver steering reaction time to abrupt-onset crosswinds, as measured in a moving-base driving simulator, Human Factors, 25(1), 103-116 